\title{
Prognostic value of the chest X-ray in patients hospitalised for heart failure
}

\author{
Daniel Pan ${ }^{1,2}$ (1) Pierpaolo Pellicori ${ }^{3} \cdot$ Karen Dobbs $^{2} \cdot$ Jeanne Bulemfu $^{2} \cdot$ Ioanna Sokoreli $^{4} \cdot$ Alessia Urbinati $^{2}$. \\ Oliver Brown ${ }^{2} \cdot$ Shirley Sze ${ }^{2,5} \cdot$ Alan S. Rigby ${ }^{6,7} \cdot$ Syed Kazmi ${ }^{2,6,7}$. Jarno M. Riistama ${ }^{8} \cdot$ John G. F. Cleland ${ }^{2,3}$. \\ Andrew L. Clark ${ }^{2,6,7}$
}

Received: 28 October 2020 / Accepted: 3 March 2021 / Published online: 22 March 2021

(c) The Author(s) 2021

\begin{abstract}
Background Patients admitted to hospital with heart failure will have had a chest X-ray (CXR), but little is known about their prognostic significance. We aimed to report the prevalence and prognostic value of the initial chest radiograph findings in patients admitted to hospital with heart failure (acute heart failure, AHF).

Methods The erect CXRs of all patients admitted with AHF between October 2012 and November 2016 were reviewed for pulmonary venous congestion, Kerley B lines, pleural effusions and alveolar oedema. Film projection (whether anterior-posterior [AP] or posterior-anterior [PA]) and cardiothoracic ratio (CTR) were also recorded. Trial registration: ISRCTN96643197 Results Of 1145 patients enrolled, 975 [median (interquartile range) age 77 (68-83) years, 61\% with moderate, or worse, left ventricular systolic dysfunction, and median NT-proBNP $5047(2337-10,945) \mathrm{ng} / \mathrm{l}] \mathrm{had}$ an adequate initial radiograph, of which 691 (71\%) were AP. The median CTR was 0.57 (IQR 0.53-0.61) in PA films and $0.60(0.55-0.64)$ in AP films. Pulmonary venous congestion was present in 756 (78\%) of films, Kerley B lines in 688 (71\%), pleural effusions in $649(67 \%)$ and alveolar oedema in $622(64 \%)$.

A CXR score was constructed using the above features. Increasing score was associated with increasing age, urea, NTproBNP, and decreasing systolic blood pressure, haemoglobin and albumin; and with all-cause mortality on multivariable analysis (hazard ratio $1.10,95 \%$ confidence intervals $1.07-1.13, p<0.001$ ).

Conclusions Radiographic evidence of congestion on a CXR is very common in patients with AHF and is associated with other clinical measures of worse prognosis.
\end{abstract}

Daniel Pan

Daniel.pan@nhs.net

1 Department of Respiratory Sciences, University of Leicester, University Road, Leicester LE1 7RH, UK

2 Castle Hill Hospital, Castle Road, Cottingham HU16 5JQ, UK

3 Robertson Centre for Biostatistics, Boyd Orr Building, University of Glasgow, Glasgow G12 8QQ, UK

4 Philips Research, High Tech, Campus 34, 5656 AE Eindhoven, The Netherlands

5 Department of Cardiovascular Sciences, University of Leicester, University Road, Leicester LE1 7RH, UK

6 Hull York Medical School, University of York, John Hughlings Jackson Building, University Road, Heslington YO10 5DD, York, UK

7 University of Hull, Cottingham Road, Hull HU6 7RX, UK

8 Philips Image Guided Therapy, Veenpluis 4-6, 5684 PC Best, The Netherlands 


\section{Graphic abstract}

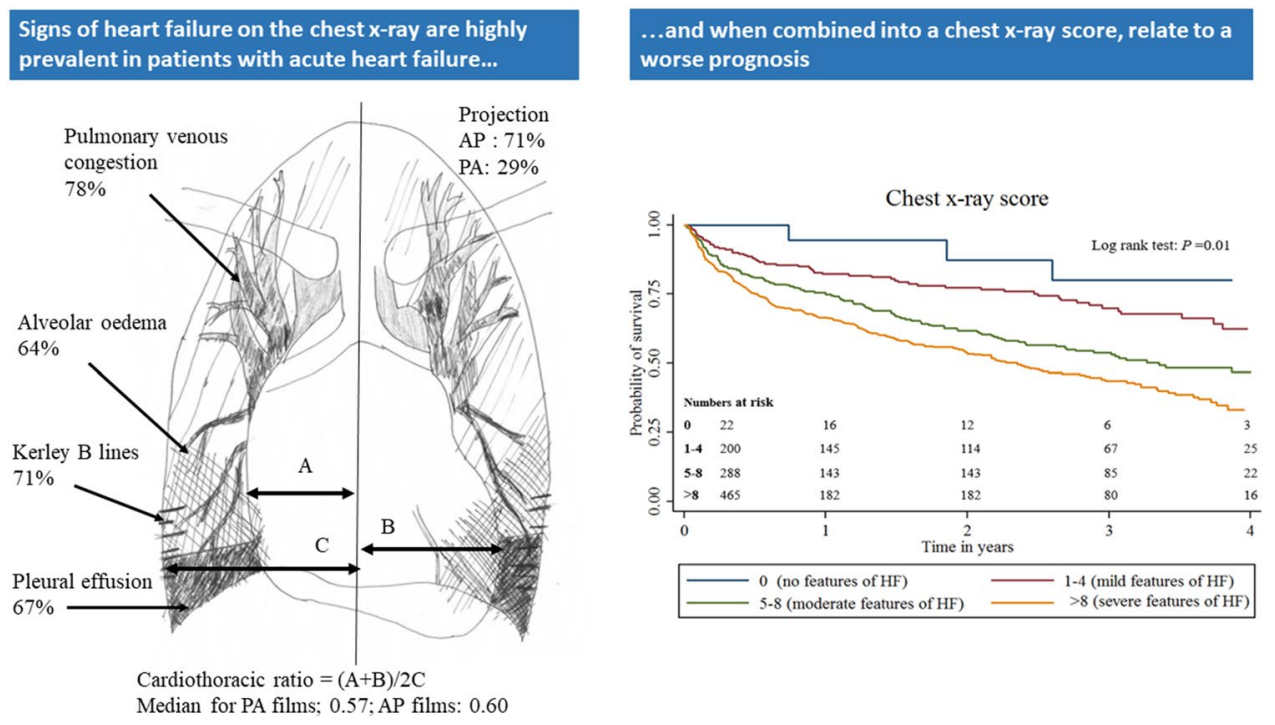

Signs of heart failure are highly prevalent in patients presenting to hospital with acute heart failure and when combined into a chest x-ray score, relate to a worse long term risk of death

Keywords Chest radiograph $\cdot$ Acute heart failure $\cdot$ Mortality $\cdot$ Congestion

\section{Introduction}

Heart failure (HF) is the leading cause of hospital admission in people aged 65 years or older in developed countries [1]. The chest X-ray (CXR) is one of the core investigations of breathless patients [2, 3]. In 1917, Danzer first identified cardiomegaly as a possible indicator of left ventricular dilation [4]. As hydrostatic pressure increases in the lungs, signs of pulmonary congestion start to appear-pulmonary venous congestion, interstitial oedema, alveolar oedema and pleural effusions - that are often used as entry criteria in trials of therapies for patients presenting with acute HF $[5,6]$.

The CXR is not a diagnostic test for HF. There is little relation between cardiothoracic ratio (CTR) and left ventricular systolic function [7]. Not all patients with acute HF have pulmonary congestion on their CXR [8]. However, amongst patients presenting to hospital with acute myocardial infarction, worsening features of HF on the CXR are related to increasing risk of death [9-13].

Fifty years ago, when rheumatic heart disease was common, acute pulmonary oedema was dramatic and life threatening [14]. Today, most patients admitted to hospital with HF no longer have new acute pulmonary oedema but instead present with decompensation of chronic underlying ventricular dysfunction, as a consequence of gradual but progressive increases in cardiac filling pressures $[15,16]$. We, therefore, described the appearance of the initial CXR in a modern cohort of patients hospitalised for HF and assessed the relation between CXR appearance and outcome. We constructed a CXR scoring system and assessed the relation between the score and outcome in addition to clinical variables.

\section{Methods}

\section{Study setting}

The observational study to predict readmission for heart failure patients (OPERA-HF) is a prospective observational study which enrolled patients hospitalised for HF in the Hull University Hospitals NHS Trust, UK. Patients had to fulfil the following criteria to be included: age $>18$ years; usual residence in the region served by the Hull University Hospitals NHS Trust; hospitalisation with HF; treatment with loop diuretics; and at least one of the following; left ventricular ejection fraction $\leq 40 \%$; left atrial dimension $\geq 4.0 \mathrm{~cm}$ [17], or N-terminal pro-B-type natriuretic peptide (NTproBNP) $>400 \mathrm{pg} / \mathrm{mL}$ (if in sinus rhythm) or $>1200 \mathrm{pg} / \mathrm{mL}$ (if in atrial fibrillation-AF) [18]. Patients who were unable to understand and comply with the protocol or unable or unwilling to give informed consent were not included in the study. 


\section{Chest radiography}

The flow of patients through the study is shown in Fig. 1. The position (supine or erect) and projection of each film is written physically on the X-ray. In the present analysis, we included all patients who had an erect chest radiograph. The patient's first radiograph on admission was reviewed. We excluded patients who had had only a supine film (as fluid in the lungs and pleura might have been distributed differently) or in whom the film was too rotated for interpretation. One investigator (Daniel Pan, DP) retrospectively reviewed all CXRs, grading the presence and severity of potential features of HF, blind to all other clinical data.

The approach to grading the CXR is illustrated in Fig. 2. The following were recorded for each patient:

- The film projection-whether posterior-anterior (PA) or anterior-posterior (AP);

\section{Enrolled in OPERA-HF: 1145 patients}

No CXR during admission: 70 patients

Remaining: 1075 patients

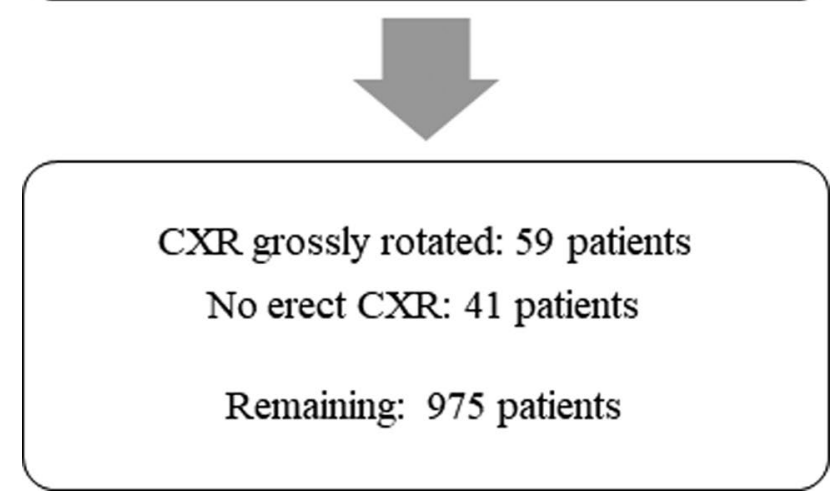

Fig. 1 Flowchart illustrating the flow of patients through the study. $C X R$ chest $\mathrm{X}$-ray, $C T$ computed tomography
- Alveolar oedema-graded as absent, present (oedema covering any of the lungs, but not all zones in both lungs) or severe (oedema covering all zones in both lungs).

- Kerley B lines-graded as the presence or absence of prominent interstitial fissure lines in and between the lung lobes.

- Pleural effusions-graded as present or not present. Presence is when there are both costophrenic and cardiophrenic blunting of one lung field.

- Pulmonary venous congestion-graded as present or absent. Presence is when there is an increase in the proportion of vessels in the upper lung zones compared to the lower lung zones.

- Heart size-measured as CTR.

The CTR was calculated using the original method described by Danzer [4]. A single line is drawn dividing the cardiac silhouette vertically through the midline. Measurement ' $A$ ' is widest point from the right heart border to the line; measurement ' $\mathrm{B}$ ' is the widest point from the left heart border to the line. Measurement ' $\mathrm{C}$ ' is the widest measurement from the midline to the inner borders of the ribs. Thus, the CTR is defined as $(\mathrm{A}+\mathrm{B}) / 2 \mathrm{C}$. An unmeasurable CTR was recorded where the cardiac borders were too obscure for accurate measurement. Although modern dictum suggests that accurate CTR interpretation is not possible in AP films, we considered a CTR of $>0.55$ to be cardiomegaly regardless of projection, in keeping with the previous literature [19].

Signs related to other pathology as routinely reported by the radiologist were also recorded. To assess repeatability of findings, 25 CXRs were randomly selected and scored by a second investigator (Shirley Sze, SS).

Patients were followed until 1st of May 2017; the main outcome of interest was all-cause mortality.

The study had ethical approval from the South Yorkshire Research Ethics Committee (REC ref: 12/YH/0344) and is conducted in accordance with ICH-GCP, Declaration of Helsinki, the Data Protection Act 1998 and NHS Act 2006.

\section{Statistical analysis}

Continuous variables are expressed as median and interquartile range (IQR). Variables that were not normally distributed were log-transformed before analysis in further models. Categorical variables are expressed as numbers and percentages (\%). Missing data were excluded from statistical analysis. Pearson's Chi-squared test and Fisher's exact row test were used to compare categorical variables between groups. Student's $t$ test and the Kruskal-Wallis test were used to compare continuous variables between the groups depending on the normality of the distribution. 


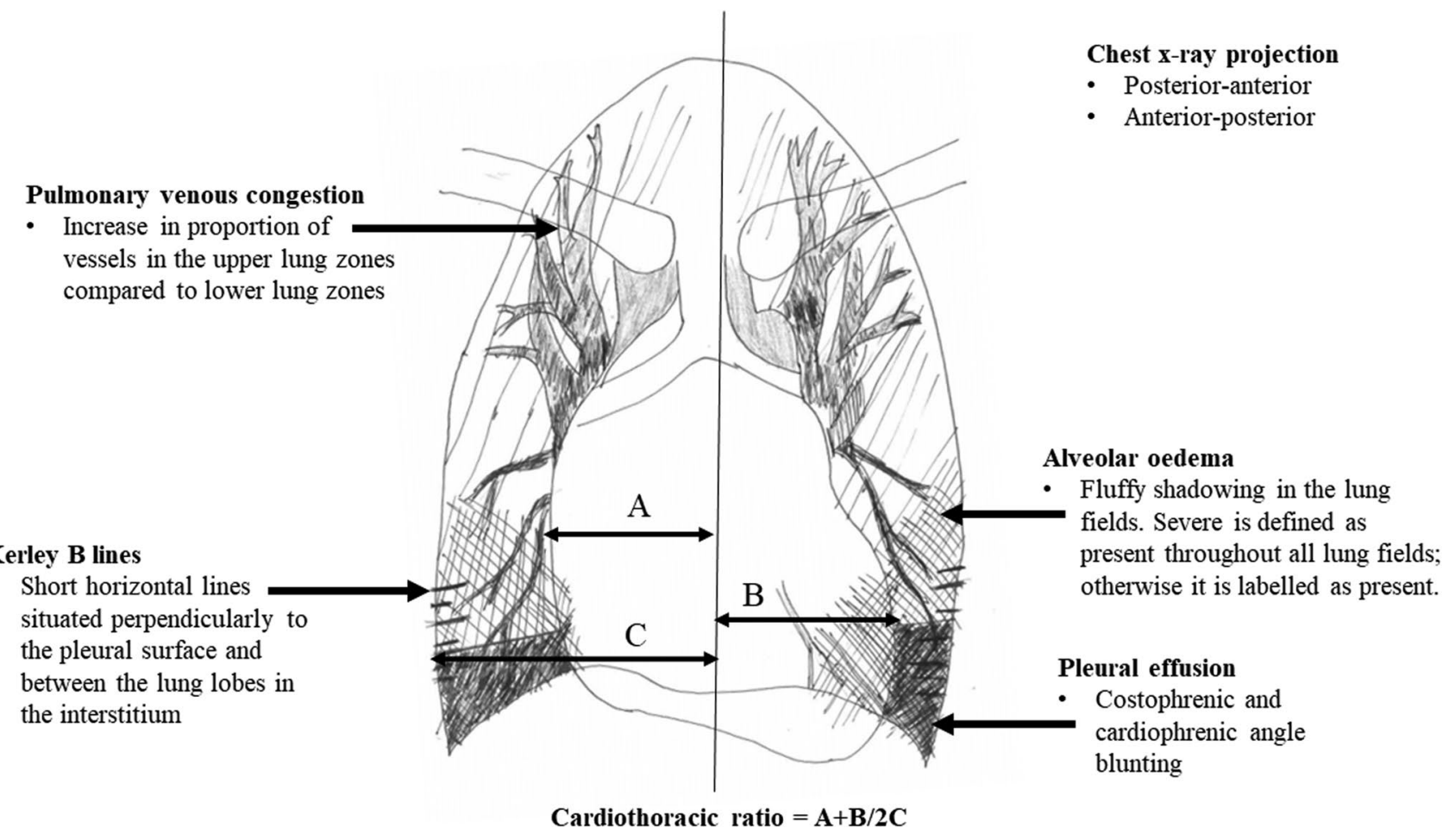

Fig. 2 Illustration demonstrating how chest X-ray features of heart failure was identified

Several Cox proportional hazard regression models were used to investigate the relation between variables and all-cause mortality. A first model was used to assign a weighting to the CXR variables based on their relation to prognosis. Only CXR variables were included in this model. Those significantly related to mortality on univariable analysis (a liberal threshold of $P<0.10$ was used) were chosen for the multivariable model. The assigned points of the CXR score were generated by the beta coefficient (log hazard ratio) for each variable from the multivariable analysis, multiplied by ten and rounded to the nearest whole number [20].

A base model was constructed to assess the incremental value of the new CXR score in addition to other important clinical variables in predicting mortality. The base model included variables which had few missing data $(<1 \%)$ and which were also significantly related to mortality on univariable Cox regression analysis (again, a liberal threshold of $P<0.10$ was used).

The individual CXR variables and the CXR score were then added to the base model, separately and in combination, to the find the best model to predict mortality. The new models' cumulative discrimination was measured using Harrell's C statistic; its statistical significance in comparison to the base model was assessed with the likelihood ratio test. A 2 -sided $P$ value $\leq 0.05$ was considered statistically significant.
Venn diagrams are used to illustrate the relation between different features of pulmonary congestion. Kaplan-Meier curves, censored at 4 years, with the log-rank statistic were used to illustrate outcome.

Repeatability of CXR findings was tested using weighted Cohen's Kappa statistic and Bland-Altman plots [21].

All analyses were performed using STATA software (version 14.2, StataCorp, Texas, United States) and Excel version 2016 (Microsoft, Redmond, United States).

\section{Results}

\section{Participants}

Between October 2012 and November 2016, 1145 patients were enrolled into OPERA-HF. After excluding 70 patients because they did not have a CXR during admission and another 100 patients because the CXRs were inadequate for interpretation, 975 remained (Fig. 1).

Table 1 shows the clinical and laboratory findings of patients recruited into the study on admission to hospital. Most patients had been hospitalised for HF in the previous year (60\%). A quarter (26\%) of patients did not have NT-proBNP measured on admission. Half of patients (54\%) presented with breathlessness on exertion; $26 \%$ with breathlessness at rest and in the remainder, a symptom other 
Table 1 Clinical and laboratory findings of patients recruited into the study on admission to hospital

\begin{tabular}{|c|c|c|}
\hline Variables & Patients & Missing \\
\hline \multicolumn{3}{|l|}{ Demographics } \\
\hline Age-years & $77(68-83)$ & 0 \\
\hline Males & $598(61 \%)$ & \\
\hline Hosp. for heart failure in the previous year & $581(60 \%)$ & \\
\hline Prior myocardial infarction & $220(23 \%)$ & \\
\hline Prior coronary artery bypass graft & $136(14 \%)$ & \\
\hline Malignancy & $108(11 \%)$ & \\
\hline Diabetes & $323(33 \%)$ & \\
\hline Chronic obstructive pulmonary disease & $160(16 \%)$ & \\
\hline ACS on admission for heart failure & $101(11 \%)$ & \\
\hline Systolic blood pressure- $\mathrm{mmHg}$ & $129(114-147)$ & \\
\hline Diastolic blood pressure- $-\mathrm{mmHg}$ & $74(62-86)$ & \\
\hline \multicolumn{3}{|l|}{ Main presenting symptom } \\
\hline Breathlessness at rest & $257(26 \%)$ & 0 \\
\hline Worsening breathlessness on exertion & $529(54 \%)$ & \\
\hline Breathlessness not a main presenting symptom & $189(19 \%)$ & \\
\hline \multicolumn{3}{|l|}{ Findings on electrocardiogram } \\
\hline Atrial fibrillation & $460(47 \%)$ & 28 \\
\hline QRS duration—milliseconds & $104(91-136)$ & \\
\hline Heart rate-beats per minute & $91(73-112)$ & \\
\hline \multicolumn{3}{|c|}{ Left ventricular systolic dysfunction on echocardiography } \\
\hline $\begin{array}{l}\text { None } \\
\text { Mild } \\
\text { Moderate } \\
\text { Severe }\end{array}$ & $\begin{array}{l}233(25 \%) \\
125(14 \%) \\
218(24 \%) \\
335(37 \%)\end{array}$ & 64 \\
\hline \multicolumn{3}{|l|}{ Blood tests } \\
\hline Sodium—mmol/L & $137(134-139)$ & 0 \\
\hline Potassium—mmol/L & $4.3(4.0-4.7)$ & 3 \\
\hline Urea-mmol/L & $8.7(6.2-13.0)$ & 0 \\
\hline Creatinine $-\mu \mathrm{mol} / \mathrm{L}$ & $104(82-144)$ & 0 \\
\hline Troponin $\mathrm{T}-\mathrm{ng} / \mathrm{ml}$ & $50(29-148)$ & 542 \\
\hline NT-proBNP-pg/ml & $5047(2337-10,945)$ & 253 \\
\hline Haemoglobin-mmol/L & $125(110-139)$ & 0 \\
\hline Chloride- $\mathrm{mmol} / \mathrm{L}$ & $101(98-105)$ & \\
\hline Albumin-mmol/L & $34(31-37)$ & 2 \\
\hline
\end{tabular}

28 patients had missing electrocardiograms because they could not be found in the clinical notes, 64 patients had missing echocardiograms because they had died before the investigation could be performed and 3 serum potassiums were missing because the blood samples had haemolysed

$Q R S$ duration-duration of QRS complex on electrocardiogram

NT-proBNP N-terminal-pro brain natriuretic peptide

Continuous variables are displayed as median (interquartile range) and categorical variables are displayed as number (percentage) than breathlessness was the reason for admission. $11 \%$ of patients presented with HF associated with an acute coronary syndrome.

\section{Chest radiography appearances}

Table 2 shows the CXR appearances of patients in the study. Most patients had AP CXRs $(71 \%$,). There was a low prevalence of features on the CXR unrelated to HF, including: consolidation $(N=91)$; pulmonary fibrosis $(N=16)$; hiatus hernia $(N=3)$; tumours/nodules $(N=7)$; pulmonary infarction $(N=5)$; pulmonary plaques $(N=13)$ and pneumonectomy $(N=1)$.

Cardiomegaly was present in $67 \%$ and un-measureable in $6 \%$. The median CTR (excluding those in whom a measurement was not possible, $N=55$ ) was 0.57 (IQR 0.53-0.61) 
Table 2 Radiological findings of patients recruited into the study on admission, and in-hospital mortality, 30-day mortality and readmission to hospital within 30 days of discharge

\begin{tabular}{lc}
\hline Variables & Patients \\
\hline Chest X-ray findings & \\
Cardiothoracic ratio $(\leq 0.55)$ & $261(27 \%)$ \\
Cardiothoracic ratio $(0.55-0.70)$ & $606(62 \%)$ \\
Cardiothoracic ratio $(>0.70)$ & $53(5 \%)$ \\
Cardiothoracic ratio: unmeasurable & $55(6 \%)$ \\
Cardiothoracic ratio & $0.59(0.55-0.64)$ \\
Film projection-anterior-posterior & $691(71 \%)$ \\
Cardiothoracic ratio (posterior-anterior films) & $0.57(0.53-0.61)$ \\
Cardiothoracic ratio (anterior-posterior films) & $0.60(0.55-0.64)$ \\
Moderate alveolar oedema & $525(54 \%)$ \\
Severe alveolar oedema & $97(10 \%)$ \\
Kerley B lines & $688(71 \%)$ \\
Pleural effusion & $649(67 \%)$ \\
Pulmonary venous congestion & $756(78 \%)$ \\
Outcomes $n=(\%)$ & \\
In-hospital mortality & $41(4 \%)$ \\
30-day mortality & $44(5 \%)$ \\
Readmission to hospital within 30-days of & $181(19 \%)$ \\
discharge & $440(45 \%)$ \\
All-cause mortality at end of follow-up &
\end{tabular}

in those with PA films and 0.60 (IQR 0.55-0.64) in those with AP films. Pulmonary venous congestion was present in $78 \%$, and a degree of alveolar oedema was present in $64 \%$ (although only $10 \%$ had severe alveolar oedema). Only one film had a pleural effusion that covered most of one lung field.

Figure 3 illustrates the percentage of patients with different features of pulmonary congestion. A third (33\%) of patients had all four features of pulmonary congestion (Fig. 3); only 5\% had none and $10 \%$ had only one. Features of pulmonary congestion were generally more common with increasing CTR and on AP compared to PA films as shown by the bar charts illustrating the proportion of patients with each feature of pulmonary congestion, by cardiothoracic ratio and chest X-ray projection in Fig. 4.

\section{Generation of the CXR score, relation to clinical variables and relation to outcome}

After a median follow-up of 671 days, 440 (45\%) patients had died of which 41 died during the index admission. A fifth $(19 \%)$ of the patients was readmitted with worsening HF within 30 days of discharge from their index hospitalisation.

Table 3 shows Cox regression analyses of chest X-ray variables and formation of the chest X-ray score; Fig. 5 shows Kaplan-Meier curves of all-cause mortality for chest X-ray projection, cardiothoracic ratio, pleural effusion, alveolar oedema. In univariable Cox regression analysis, each radiological abnormality, except pulmonary venous congestion, was associated with all-cause mortality. Consequently, a score was constructed using all CXR signs apart from pulmonary venous congestion.

Table 4 shows clinical and laboratory findings of patients recruited into the study and in-hospital mortality, 30-day mortality and readmission to hospital within 30 days of discharge by increasing chest X-ray score. There was no relation between the CXR score and the presence (or absence) of left ventricular systolic dysfunction. Although associated with all-cause mortality at 4 years, the CXR score was neither associated with in-hospital mortality, nor mortality or readmission within 30 days of discharge. A worsening CXR score was associated with older age, higher serum
Fig. 3 Venn diagram illustrating percentage of patients with different features of pulmonary congestion

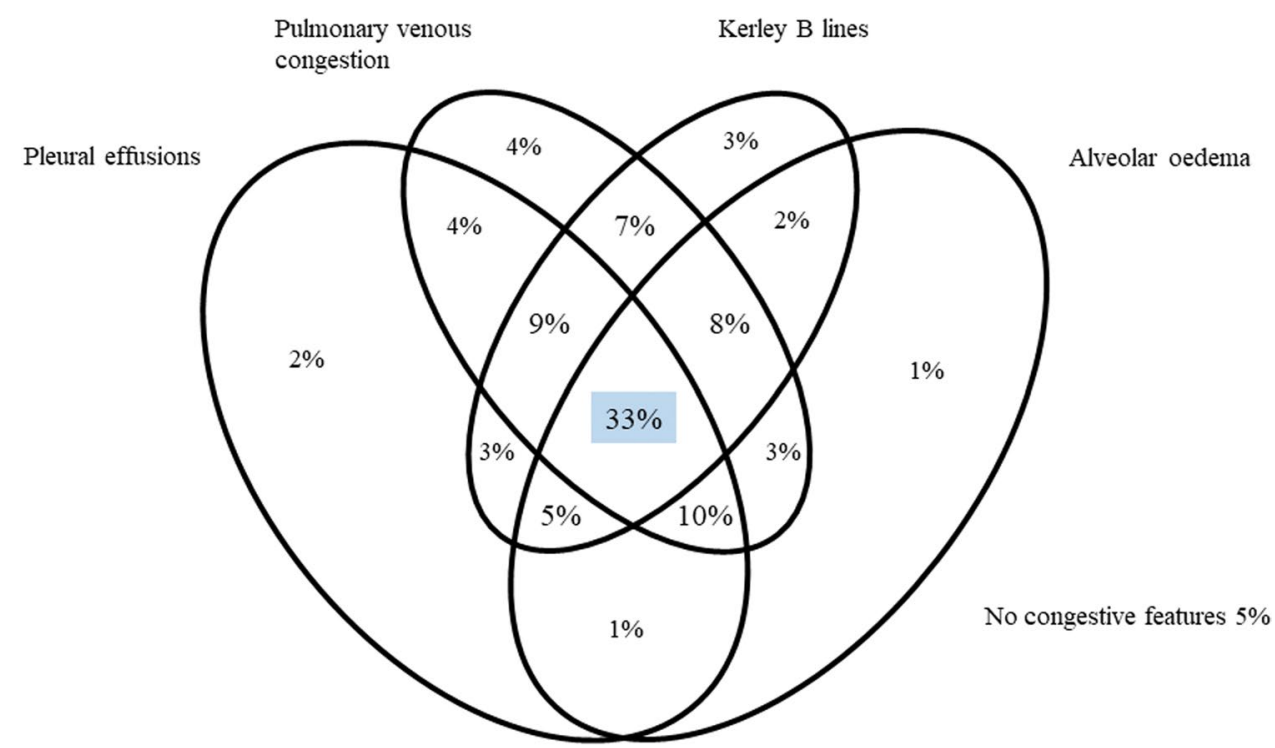


$\leq 0.55$

$0.55-0.70$

$>0.70$ or unmeasurable
Posterior-anterior

Anterior-posterior
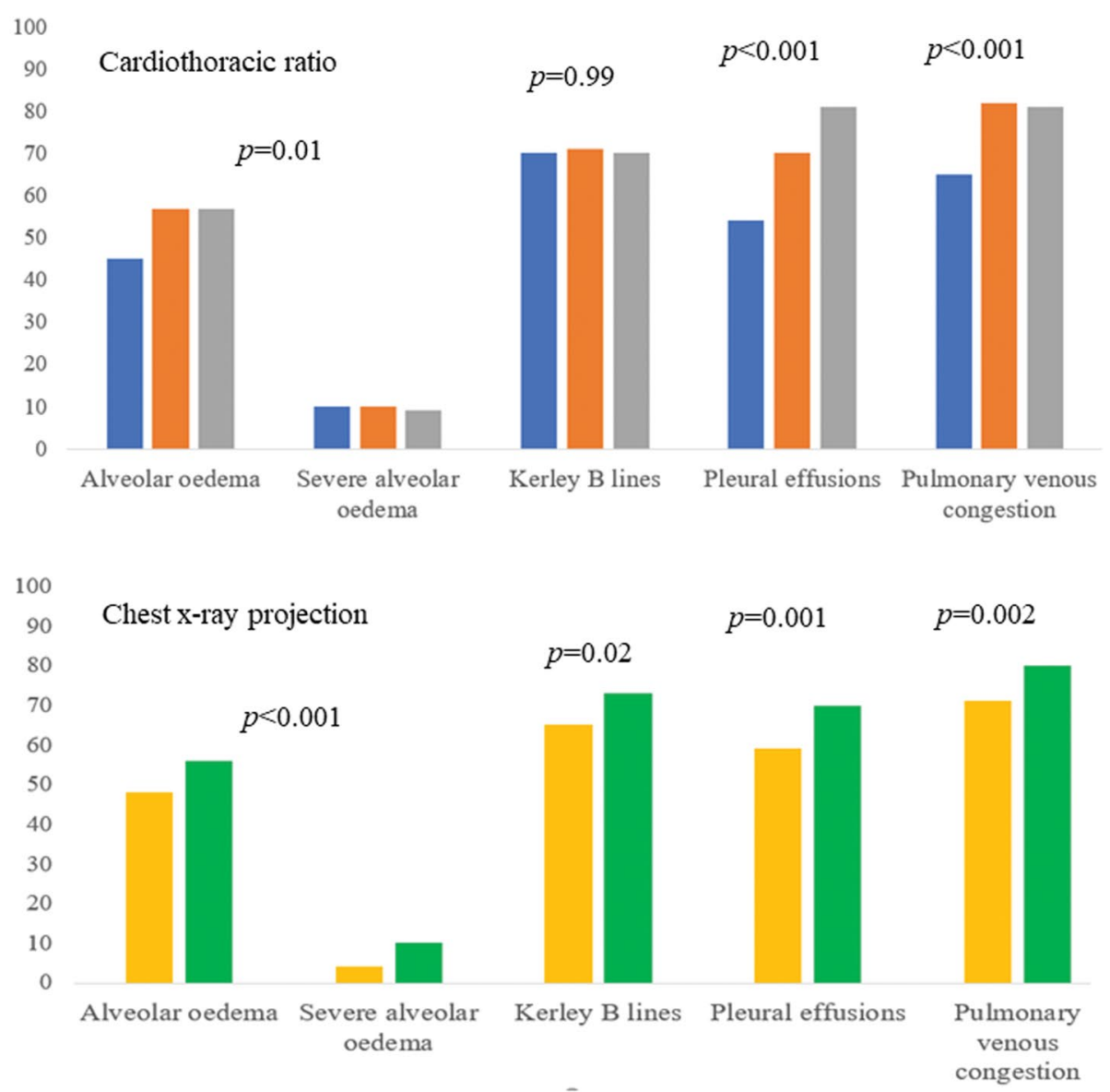

Fig. 4 Bar charts illustrating proportion of patients with each feature of pulmonary congestion, by cardiothoracic ratio and chest X-ray projection. Pearson's Chi-squared test was used

concentrations of potassium and urea and higher plasma NT-proBNP (especially if the patient was in AF), lower values for diastolic blood pressure, haemoglobin, and albumin, being a woman, prior CABG and presenting with breathlessness as the main symptom.

CXR scores were lower in the $5 \%$ of patients $(n=49)$ whose first radiograph was taken more than $24 \mathrm{~h}$ after admission than in those who had their CXR earlier [median score 3 (IQR 3-7) v 7 (5-9), $P<0.001]$.

\section{Relation of the CXR score to prognosis in addition to other variables}

Tables 5 and 6 show univariable and multivariable Cox regression analyses of clinical and laboratory variables and the chest $\mathrm{X}$-ray score On univariable Cox regression analysis, age, gender, hospitalisation for heart failure in the previous year, prior MI or CABG, malignancy, lower systolic and diastolic blood pressures, lower heart rate, lower sodium, haemoglobin, chloride and albumin and higher potassium, renal function, troponin, and NT-proBNP, and a higher CXR score was related to mortality. In a multivariable model, older age and higher serum concentrations of urea and sodium, lower systolic blood pressure, lower heart rate and lower serum concentrations of chloride and albumin were independent predictors of mortality. The CXR score was also independently associated with mortality (HR $1.10,95 \%$ CI $1.07-1.13$ per point increase, $P<0.001)$. However, CXR score did not improve the model's $c$-statistic for predicting mortality at different time-points as shown in Table 7.

\section{Reproducibility}

CXR variables varied in reliability, with alveolar oedema being the most reliable and CTR being the least, as shown in Table 7. 
Table 3 Univariable and multivariable Cox regression analyses of chest X-ray variables and formation of the chest X-ray score

\begin{tabular}{|c|c|c|c|c|c|c|}
\hline Outcome: all-cause mortality & Hazard ratio (95\% CI) & Wald $X^{2}$ & $P$ value & Hazard ratio $(95 \% \mathrm{CI})$ & $\beta$-coefficient & Score \\
\hline \multicolumn{7}{|l|}{ Alveolar oedema } \\
\hline Absent & Referent & Referent & Referent & Referent & Referent & 0 \\
\hline Present & $1.20(0.98-1.47)$ & 1.71 & 0.09 & $1.04(0.84-1.31)$ & 0.05 & 1 \\
\hline Severe & $1.67(1.22-2.28)$ & 3.20 & 0.001 & $1.35(0.97-1.88)$ & 0.30 & 3 \\
\hline \multicolumn{7}{|l|}{ Kerley B lines } \\
\hline Absent & Referent & Referent & Referent & Referent & Referent & 0 \\
\hline Present & $1.28(1.04-1.59)$ & 2.30 & 0.02 & $1.21(0.98-1.51)$ & 0.19 & 2 \\
\hline \multicolumn{7}{|l|}{ Cardiothoracic ratio } \\
\hline$\leq 0.55$ & Referent & Referent & Referent & Referent & Referent & 0 \\
\hline $0.55-0.70$ & $1.21(0.96-1.52)$ & 1.62 & 0.10 & $1.12(0.89-1.41)$ & 0.12 & 1 \\
\hline$>0.70 /$ unmeasurable & $1.84(1.35-2.50)$ & 3.84 & $<0.001$ & $1.60(1.16-2.19)$ & 0.46 & 5 \\
\hline \multicolumn{7}{|l|}{ Chest X-ray projection } \\
\hline Posterior-anterior & Referent & Referent & Referent & Referent & Referent & 0 \\
\hline Anterior-posterior & $1.13(1.07-1.20)$ & 4.28 & $<0.001$ & $1.47(1.17-1.86)$ & 0.38 & 4 \\
\hline \multicolumn{7}{|l|}{ Pleural effusions } \\
\hline Absent & Referent & Referent & Referent & Referent & Referent & 0 \\
\hline Present & $1.29(1.05-1.59)$ & 2.47 & 0.01 & $1.12(0.89-1.40)$ & 0.11 & 1 \\
\hline \multicolumn{7}{|l|}{ Pulmonary venous congestion } \\
\hline Absent & Referent & Referent & Referent & N/A & N/A & N/A \\
\hline Present & $1.06(0.85-1.33)$ & 0.53 & 0.60 & N/A & N/A & N/A \\
\hline \multicolumn{7}{|l|}{ Chest X-ray score } \\
\hline Chest X-ray score & $1.10(1.07-1.13)$ & 6.15 & $<0.001$ & N/A & N/A & N/A \\
\hline
\end{tabular}

The score was constructed using the beta-coefficients (log hazard ratio) of a multivariable model, containing only chest X-ray variables that were significantly related to all-cause mortality on univariable analysis $(p<0.1)$. These variables were: alveolar oedema, Kerley B lines, cardiothoracic ratio, chest X-ray projection and pleural effusions. For example, the beta coefficient for Kerley B lines from the multivariate analysis is 0.19 - which was rounded to 0.2 multiplied by 100 to give 2 points

CI confidence intervals, N/A not applicable

\section{Discussion}

There are three main findings from this study. First, radiological evidence of congestion is very common in patients presenting to hospital with HF. Second, patients presenting with breathlessness as their dominant symptom have a higher CXR score. Third, increasing pulmonary congestion on the CXR score is related to worsening HF as assessed by other clinical measures such as age, potassium, urea, NT-proBNP, haemoglobin and albumin, and is associated with increasing all-cause mortality, but not related to overall prognosis when these other variables are taken into account.

We found that the commonest abnormal feature on the chest X-ray was venous congestion, presumably representing a rise in left atrial pressure. As heart failure worsens, fluid can escape from pulmonary capillaries into the interstitial and pleural spaces resulting in Kerley B lines and pleural effusions, the next two most common features we found. Frank alveolar oedema was the least common finding, and represents the patient passing a 'tipping point' when the capacity of the lymphatics to remove fluid is exceeded and fluid starts to accumulate in the airspaces of the lungs [16].
Previous older studies have graded congestion in an incremental fashion based on the aforementioned sequence of events, with stage one being pulmonary venous congestion, stage two interstitial oedema and stage three alveolar oedema [11-13, 22]. We chose to grade all features separately and found that although many had pulmonary venous congestion and few had severe alveolar oedema, patients commonly have features of pulmonary congestion not in sequence. It may be that if pulmonary congestion happens very rapidly, the radiographic features might not occur in order.

Our study findings confirm that the CXR can identify pulmonary congestion as a potential cause of breathlessness in patients presenting to hospital. Since congestion on the CXR is still related to mortality in HF patients on univariable analysis, this should prompt clinicians to adequately diurese the patient when necessary.

Only a small proportion of patients $(91 / 975,9 \%)$ in our cohort had evidence of pneumonia on the CXR. Future studies should investigate whether the CXR can help to differentiate pulmonary congestion from other causes of breathlessness, and influence therapeutic decision making beyond HF, such as the initiation of antibiotics. The CXR 
(a)

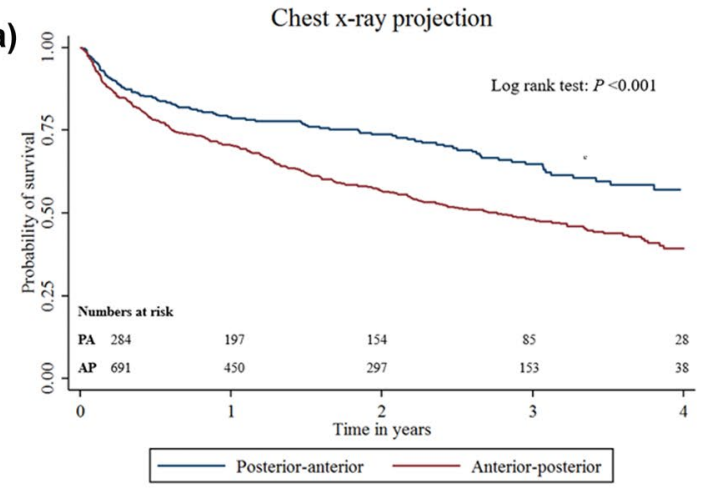

(b)

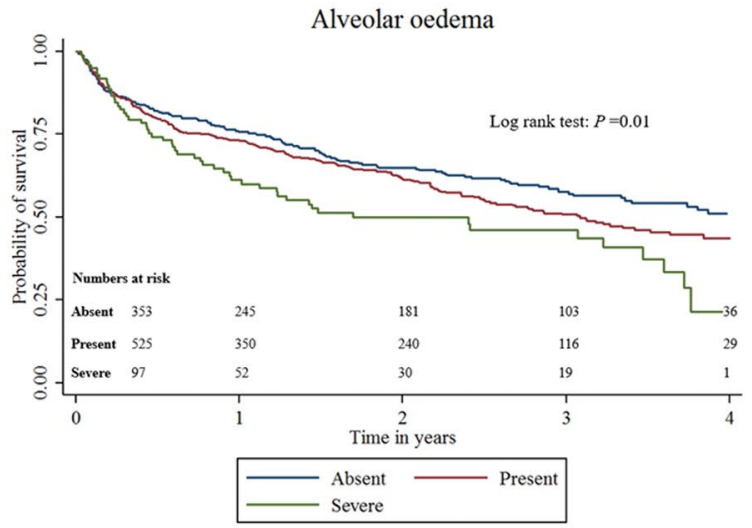

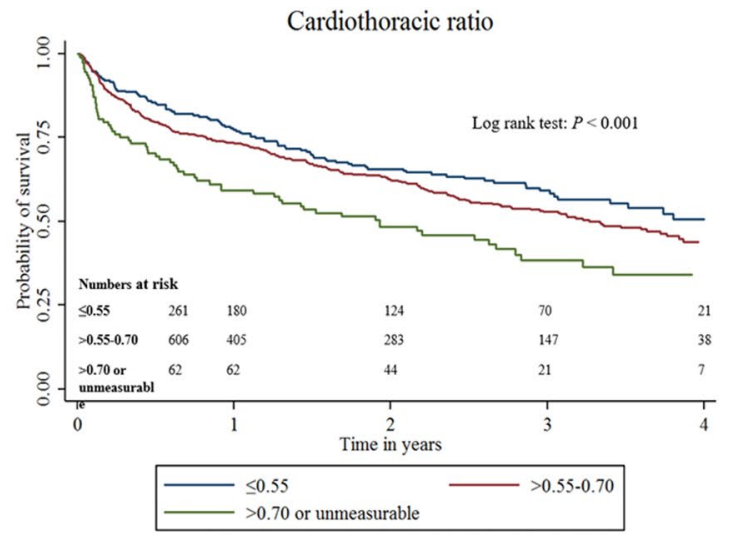

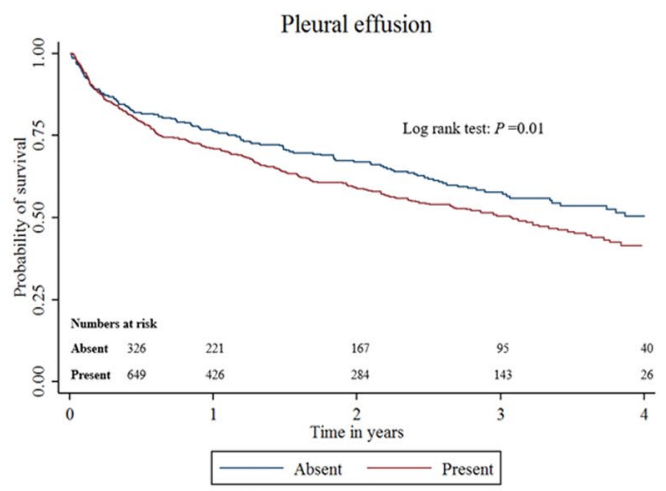

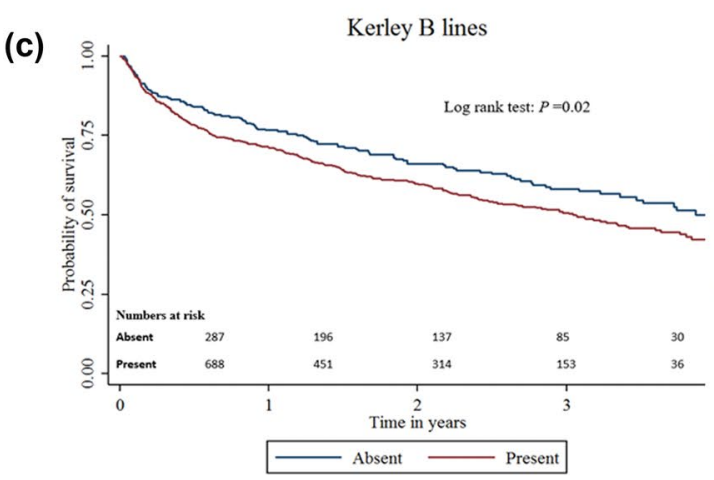

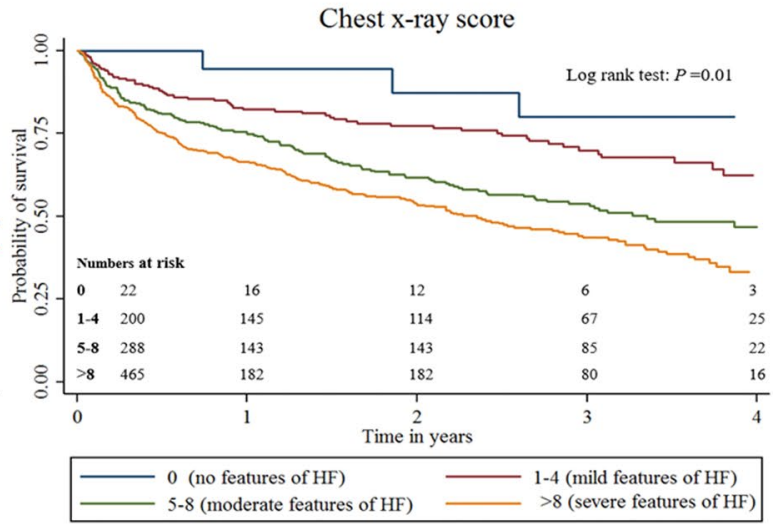

Fig. 5 Kaplan-Meier curves of all-cause mortality for chest X-ray projection, cardiothoracic ratio, pleural effusion, alveolar oedema, Kerley B lines and the chest $\mathrm{X}$-ray score. $A P$ anterior-posterior, $P A$ posterior-anterior, $H F$ heart failure

remains a source of important clinical information and may be used within artificial intelligence algorithms, to help assist clinicians improve the diagnosis of AHF and guide management [23].

To our knowledge, this is the first study which has found a relation between CXR projection and all-cause mortality. If a patient is too unwell to stand, inhale and hold their breath, then an AP film in the sitting upright position is performed [24], and thus projection is a marker of a patient's fitness during their acute illness.

Our findings agree with previous work, which found that cardiomegaly on the CXR, regardless of projection, was related to a worse prognosis in patients with acute and chronic HF [9, 10]. However, despite a high level of readmission to hospital within 30 days of discharge, in-hospital and 30-day mortality was low. None of these outcomes 
Table 4 Clinical and laboratory findings of patients recruited into the study and in-hospital mortality, 30-day mortality and readmission to hospital within 30 days of discharge by increasing chest X-ray score

\begin{tabular}{|c|c|c|c|c|c|}
\hline \multirow{2}{*}{$\frac{\text { Variables }}{\text { Demographics }}$} & \multicolumn{4}{|c|}{ Chest X-ray score } & \multirow[t]{2}{*}{$P$ value } \\
\hline & & & & & \\
\hline Chest X-ray score $N=975$ & $\begin{array}{l}0 \\
N=22(2 \%)\end{array}$ & $\begin{array}{l}1-4 \\
N=200(20 \%)\end{array}$ & $\begin{array}{l}5-8 \\
N=288(30 \%)\end{array}$ & $\begin{array}{l}>8 \\
N=465(48 \%)\end{array}$ & \\
\hline Age-years & $74(67-78)$ & $73(64-80)$ & $76(67-83)$ & $78(71-85)$ & $<0.001$ \\
\hline Men & $15(68 \%)$ & $148(74 \%)$ & $176(61 \%)$ & $259(56 \%)$ & $<0.001$ \\
\hline Hosp. for heart failure in the previous year & $13(59 \%)$ & $111(56 \%)$ & $180(63 \%)$ & $277(60 \%)$ & 0.49 \\
\hline Prior myocardial infarction & $6(27 \%)$ & $40(20 \%)$ & $64(22 \%)$ & $110(24 \%)$ & 0.71 \\
\hline Prior coronary artery bypass graft & $7(32 \%)$ & $35(18 \%)$ & $39(14 \%)$ & $55(12 \%)$ & 0.02 \\
\hline Malignancy & $3(14 \%)$ & $15(8 \%)$ & $36(13 \%)$ & $54(12 \%)$ & $0.27 *$ \\
\hline Diabetes & $5(23 \%)$ & $57(29 \%)$ & $105(36 \%)$ & $156(34 \%)$ & $0.21 *$ \\
\hline Chronic obstructive pulmonary disease & $4(18 \%)$ & $27(14 \%)$ & $41(14 \%)$ & $88(19 \%)$ & $0.22 *$ \\
\hline ACS on admission for heart failure & $4(18 \%)$ & $12(6 \%)$ & $33(12 \%)$ & $52(12 \%)$ & $0.10 *$ \\
\hline Systolic blood pressure—-mmHg & $139(113-154)$ & $130(115-146)$ & $128(116-150)$ & $129(112-145)$ & 0.50 \\
\hline Diastolic blood pressure $-\mathrm{mmHg}$ & $78(65-86)$ & $79(65-89)$ & $74(63-87)$ & $71(62-85)$ & 0.01 \\
\hline \multicolumn{6}{|l|}{ Breathlessness at presentation } \\
\hline Not the main complaint & $10(46 \%)$ & $34(17 \%)$ & $61(21 \%)$ & $84(18 \%)$ & $0.04 *$ \\
\hline Worsening on exertion & $11(50 \%)$ & $113(57 \%)$ & $155(54 \%)$ & $250(54 \%)$ & \\
\hline Severe at rest & $1(5 \%)$ & $53(27 \%)$ & $72(25 \%)$ & $131(28 \%)$ & \\
\hline \multicolumn{6}{|l|}{ ECG findings } \\
\hline Atrial fibrillation & $9(41 \%)$ & $98(49 \%)$ & $128(44 \%)$ & $225(48 \%)$ & 0.63 \\
\hline QRS duration—milliseconds & $122(34 \%)$ & $113(39 \%)$ & $111(33 \%)$ & $118(45 \%)$ & 0.09 \\
\hline Heart rate—beats/minute & $87(25 \%)$ & $93(27 \%)$ & $95(31 \%)$ & $96(28 \%)$ & 0.38 \\
\hline \multicolumn{6}{|l|}{ Left ventricular systolic dysfunction } \\
\hline None-mild & $8(38 \%)$ & $70(36 \%)$ & $97(36 \%)$ & $183(43 \%)$ & 0.21 \\
\hline Moderate-severe & $13(62 \%)$ & $123(64 \%)$ & $174(64 \%)$ & $243(57 \%)$ & \\
\hline \multicolumn{6}{|l|}{ Blood tests } \\
\hline Sodium-mmol/L & $137(135-139)$ & $137(134-139)$ & $137(134-139)$ & $137(134-139)$ & 0.83 \\
\hline Potassium—mmol/L & $4.3(4.0-4.4)$ & $4.3(4.0-4.5)$ & $4.3(4.0-4.6)$ & $4.4(4.0-4.9)$ & 0.02 \\
\hline Urea-mmol/L & $9.3(5.9-11.6)$ & $8.1(6.0-11.5)$ & $\begin{array}{l}8.3 \\
(5.7-12.9)\end{array}$ & $\begin{array}{l}9.4 \\
(6.5-13.8)\end{array}$ & $0.002 * *$ \\
\hline Creatinine $-\mu \mathrm{mol} / \mathrm{L}$ & $\begin{array}{l}100 \\
(90-156)\end{array}$ & $\begin{array}{l}102 \\
(81-129)\end{array}$ & $\begin{array}{l}102 \\
(81-145)\end{array}$ & $\begin{array}{l}108 \\
(83-154)\end{array}$ & $0.08 * *$ \\
\hline Troponin $\mathrm{T}-\mathrm{ng} / \mathrm{ml}$ & $\begin{array}{l}36 \\
(25-92)\end{array}$ & $\begin{array}{l}40 \\
(26-82)\end{array}$ & $\begin{array}{l}53 \\
(30-131)\end{array}$ & $\begin{array}{l}59 \\
(31-165)\end{array}$ & $0.08 * *$ \\
\hline NT-proBNP—pg/ml & $\begin{array}{l}2079 \\
(1467-2974)\end{array}$ & $\begin{array}{l}4569 \\
(1683-10,055)\end{array}$ & $\begin{array}{l}5317 \\
(2633-10,985)\end{array}$ & $\begin{array}{l}5371 \\
(2644-11,684)\end{array}$ & $0.003 * *$ \\
\hline NT-proBNP in SR-pg/ml & $\begin{array}{l}2612 \\
(1378-7211)\end{array}$ & $\begin{array}{l}4999 \\
(1899-10,510)\end{array}$ & $\begin{array}{l}4987 \\
(2628-12,031)\end{array}$ & $\begin{array}{l}5498 \\
(2185-11,793)\end{array}$ & $0.45^{* *}$ \\
\hline NT-proBNP in AF-pg/ml & $\begin{array}{l}1826 \\
(1467-2142)\end{array}$ & $\begin{array}{l}3942 \\
(1448-9599)\end{array}$ & $\begin{array}{l}5663 \\
(2633-10,682)\end{array}$ & $\begin{array}{l}5307 \\
(3117-11,684)\end{array}$ & $0.002 * *$ \\
\hline Haemoglobin-mmol/L & $136(123-142)$ & $130(116-143)$ & $122(109-139)$ & $123(108-137)$ & 0.004 \\
\hline Chloride—-mmol/L & $103(100-105)$ & $101(98-105)$ & $101(98-105)$ & $101(97-105)$ & 0.48 \\
\hline Albumin-mmol/L & $37(34-39)$ & $34(32-38)$ & $34(31-37)$ & $34(31-37)$ & 0.002 \\
\hline \multicolumn{6}{|l|}{ Outcomes } \\
\hline In-hospital mortality & $0(0 \%)$ & $6(3 \%)$ & $11(4 \%)$ & $24(5 \%)$ & $0.57 *$ \\
\hline 30-day mortality & $0(0 \%)$ & $6(3 \%)$ & $13(5 \%)$ & $25(5 \%)$ & $0.52 *$ \\
\hline Readmission within 30 days of discharge & $3(14 \%)$ & $34(17 \%)$ & $56(20 \%)$ & $88(19 \%)$ & $0.87 *$ \\
\hline
\end{tabular}

QRS duration-duration of QRS complex on electrocardiogram, NT-proBNP N-terminal-pro brain natriuretic peptide

Continuous variables are displayed as median (interquartile range) and categorical variables are displayed as number (percentage). For categorical variables, Pearson's Chi-squared test was used. If variables did not fit the assumptions of the Pearson's Chi-squared test, Fisher's exact test was used (labelled with *). For continuous variables, student's $t$ test and ANOVA was used to compare variables that were normally distributed 
Table 4 (continued)

and Kruskal-Wallis was used for variables that were not normally distributed (labelled with $* *$ )

Table 5 Univariable Cox regression analyses of clinical and laboratory variables and the chest X-ray score

\begin{tabular}{|c|c|c|c|}
\hline \multirow[t]{2}{*}{ Outcome: all-cause mortality } & \multicolumn{3}{|l|}{ Univariable analysis } \\
\hline & Hazard ratio $(95 \% \mathrm{CI})$ & Wald $X^{2}$ & $P$ value \\
\hline \multicolumn{4}{|l|}{ Demographics } \\
\hline Age (per year increase) & $1.05(1.04-1.06)$ & 10.30 & $<0.001$ \\
\hline \multicolumn{4}{|l|}{ Gender (male vs female) } \\
\hline Hosp. for heart failure in the previous year (yes vs no) & $1.48(1.21-1.80)$ & 3.86 & $<0.001$ \\
\hline Prior myocardial infarction (yes vs no) & $1.41(1.14-1.74)$ & $3 . .22$ & 0.001 \\
\hline Prior coronary artery bypass graft (yes vs no) & $1.55(1.22-1.98)$ & 3.52 & $<0.001$ \\
\hline Malignancy (yes vs no) & $1.35(1.01-1.80)$ & 2.06 & 0.04 \\
\hline Diabetes (yes vs no) & $1.19(0.98-1.44)$ & 1.74 & 0.08 \\
\hline \multicolumn{4}{|l|}{ Chronic obstructive pulmonary disease (yes vs no) } \\
\hline \multicolumn{4}{|l|}{ ACS on admission for heart failure } \\
\hline Systolic blood pressure - mmHg (per 10 unit increase) & $0.93(0.89-0.97)$ & -3.71 & $<0.001$ \\
\hline Diastolic blood pressure - mmHg (per 10 unit increase) & $0.98(0.97-0.98)$ & -7.29 & $<0.001$ \\
\hline \multicolumn{4}{|l|}{ Main presenting symptom } \\
\hline Breathlessness not main presenting symptom & Referent & Referent & Referent \\
\hline Worsening breathlessness on exertion & $1.05(0.83-1.35)$ & 0.43 & 0.67 \\
\hline Breathlessness at rest & $0.82(0.62-1.09)$ & -1.38 & 0.17 \\
\hline \multicolumn{4}{|l|}{ Findings on ECG } \\
\hline Atrial fibrillation (yes vs no) & $1.07(0.89-1.29)$ & 1.70 & 0.49 \\
\hline QRS duration—milliseconds (per unit increase in $\log$ QRS duration) & $1.13(0.82-1.56)$ & 0.75 & 0.45 \\
\hline Heart rate—beats/second (per 10 unit increase) & $0.92(0.88-0.95)$ & -4.92 & $<0.001$ \\
\hline \multicolumn{4}{|l|}{ Left ventricular systolic dysfunction } \\
\hline None or mild & Referent & Referent & Referent \\
\hline Moderate or severe & $0.78(0.64-0.95)$ & -2.42 & 0.02 \\
\hline \multicolumn{4}{|l|}{ Blood tests } \\
\hline Sodium-mmol/L (per unit increase) & $0.97(0.95-0.99)$ & -2.64 & 0.008 \\
\hline Potassium—mmol/L (per unit increase) & $1.20(1.04-1.39)$ & 2.51 & 0.01 \\
\hline Urea-mmol/L (per unit increase in log urea) & $2.35(2.00-2.77)$ & 10.26 & $<0.001$ \\
\hline Creatinine $-\mu \mathrm{mol} / \mathrm{L}$ (per unit increase in log creatinine) & $2.33(1.90-2.86)$ & 8.06 & $<0.001$ \\
\hline Troponin—ng/ml (per unit increase in log troponin) & $1.17(1.08-1.27)$ & 3.68 & $<0.001$ \\
\hline NT-proBNP—pg/ml (per unit increase in log NT-proBNP) & $1.33(1.20-1.48)$ & 5.43 & $<0.001$ \\
\hline Haemoglobin-mmol/L (per 10 unit increase) & $0.88(0.84-0.92)$ & -6.15 & $<0.001$ \\
\hline Chloride-mmol/L (per 10 unit increase) & $0.61(0.51-0.73)$ & -5.49 & $<0.001$ \\
\hline Albumin $-\mathrm{mmol} / \mathrm{L}$ (per 10 unit increase) & $0.60(0.50-0.72)$ & -5.37 & $<0.001$ \\
\hline \multicolumn{4}{|l|}{ Chest X-ray score } \\
\hline Chest X-ray score (per unit increase) & $1.10(1.07-1.13)$ & 6.15 & $<0.001$ \\
\hline
\end{tabular}

Variables which were not normally distributed were log-transformed

$Q R S$ duration-duration of QRS complex on electrocardiogram, NT-proBNP N-terminal-pro brain natriuretic peptide, N/A not applicable

strongly related to the degree of congestion, consistent with the observation that early readmissions are often not for HF [25-27]. Our CXR score was strongly related to clinical variables that are related to the prognosis of patients with acute HF. Thus, it is not surprising that CXR features do not add much to the predictive power of models that included these variables.

One surprising finding was that a lower initial heart rate was related to higher mortality. Lourenco and colleagues had similar findings amongst 564 patients presenting with acute 
Table 6 Multivariable Cox regression analysis

\begin{tabular}{|c|c|c|c|c|c|c|}
\hline \multirow[t]{2}{*}{ Outcome: all-cause mortality } & \multicolumn{3}{|c|}{ Multivariable analysis (base model) } & \multicolumn{3}{|c|}{$\begin{array}{l}\text { Multivariable analysis (base model and } \\
\text { chest X-ray score) }\end{array}$} \\
\hline & Hazard ratio $(95 \% \mathrm{CI})$ & Wald $X^{2}$ & $P$ value & Hazard ratio $(95 \% \mathrm{CI})$ & Wald $X^{2}$ & $P$ value \\
\hline \multicolumn{7}{|l|}{ Demographics } \\
\hline Age (per year increase) & $1.04(1.03-1.05)$ & 6.95 & $<0.001$ & $1.04(1.03-1.05)$ & 6.33 & $<0.001$ \\
\hline Systolic blood pressure $-\mathrm{mmHg}$ (per 10 unit increase) & $0.94(0.89-0.99)$ & -2.52 & 0.01 & $0.94(0.90-0.99)$ & -2.40 & 0.02 \\
\hline \multicolumn{7}{|l|}{ Findings on ECG } \\
\hline Heart rate—beats/minute (per 10 unit increase) & $0.95(0.91-0.98)$ & -2.78 & 0.005 & $0.94(0.90-0.97)$ & -3.25 & 0.001 \\
\hline \multicolumn{7}{|l|}{ Blood tests } \\
\hline Sodium—mmol/L (per unit increase) & $1.05(1.01-1.08)$ & -2.55 & 0.01 & $1.04(1.01-1.08)$ & 2.33 & 0.02 \\
\hline Urea—mmol/L (per unit increase in log urea) & $1.41(1.01-1.98)$ & 2.00 & 0.05 & $1.42(1.01-1.99)$ & 2.01 & 0.04 \\
\hline Chloride—-mmol/L (per 10 unit increase) & $0.51(0.38-0.67)$ & -4.69 & $<0.001$ & $0.51(0.38-0.67)$ & -4.71 & $<0.001$ \\
\hline Albumin $-\mathrm{mmol} / \mathrm{L}$ (per 10 unit increase) & $0.69(0.54-0.88)$ & -3.03 & 0.002 & $0.71(0.56-0.91)$ & -2.72 & 0.006 \\
\hline \multicolumn{7}{|l|}{ Chest X-ray score } \\
\hline Chest X-ray score (per unit increase) & N/A & & & $1.07(1.03-1.11)$ & 3.75 & $<0.001$ \\
\hline
\end{tabular}

Only variables significantly related to prognosis $(p<0.05)$ are displayed. Variables which were not normally distributed were log-transformed. The base model contains only variables that were significantly related to all-cause mortality on univariable analysis, displayed in Table 3 QRS duration-duration of QRS complex on electrocardiogram, NT-proBNP N-terminal-pro brain natriuretic peptide, N/A not applicable

Table 7 Reproducibility statistics (Cohen's kappa for categorical variables and Bland Altman limits of agreement for continuous variables) got the chest X-ray variables and the chest X-ray score as well as the Harrell's $c$-statistic and likelihood ratio tests of the multivariable Cox regression models

\begin{tabular}{llllll}
\hline Model & $\begin{array}{l}\text { Reproducibility }(p \\
\text { value/95\% CI })\end{array}$ & $\begin{array}{l}\text { Harrell's concordance } \\
\text { statistic }(P \text { value })\end{array}$ & & \\
\hline Mortality $(N, \%)$ & & 1 month $(38,100 \%)$ & 1 year $(263,80 \%)$ & 3 years $(409,56 \%)$ & 4 years $(435,48 \%)$ \\
Base model & & 0.84 & 0.72 & 0.70 & 0.71 \\
$\quad$ + B lines & $0.55(0.001)$ & $0.84(0.42)$ & $0.72(0.55)$ & $0.70(0.90)$ & $0.71(0.76)$ \\
+ Effusions & $0.78(<0.001)$ & $0.84(0.90)$ & $0.72(0.14)$ & $0.70(0.39)$ & $0.71(0.83)$ \\
+ Oedema & $0.82(<0.001)$ & $0.85(0.10)$ & $0.72(0.05)$ & $0.70(0.18)$ & $0.71(0.30)$ \\
+ CTR & $0.53(0.003)$ & $0.84(0.65)$ & $0.72(0.01)$ & $0.70(0.02)$ & $0.71(0.01)$ \\
$\quad$ + Projection & N/A & $0.83(0.51)$ & $0.72(0.01)$ & $0.70(0.19)$ & $0.71(0.05)$ \\
+ CXR score & $0.40(-1.90-2.67)$ & $0.84(0.77)$ & $0.72(<0.001)$ & $0.70(0.01)$ & $0.71(0.006)$ \\
\hline
\end{tabular}

The base model contains variables displayed in Tables 5 and 6 and is the same base model used in this table

CI confidence intervals, N/A not applicable

HF to a single centre [28]. In both studies, the first heart rate measurement was recorded on presentation to hospital. Although a higher resting heart rate predicts higher mortality in chronic HF, a faster initial heart rate in acute HF may be a marker of a preserved autonomic nervous response.

\section{Limitations}

This is a single centre study. Most patients were of Caucasian ethnicity. Notably, Kobayashi and colleagues recently examined pulmonary congestion on the CXR in 117 patients and found that a worsening congestion score index was associated with a composite of all-cause mortality or rehospitalisation for HF at 90 days [29]. The index was generated from dividing the chest X-ray into six zones, and grading the severity of congestion in each lung zone. Our method of grading the CXR is far more likely to be the way CXRs are routinely examined in the UK. We have also taken into account radiograph projection or CTR, both of which we have shown to relate to worse prognosis.

Our version of the CXR score need to be validated in another population. Although reports from radiologists were used, no radiologists were involved in the retrospective review of the films. This may account for some of the variation in reproducibility of CXR variables and possibly misinterpretation of films, such as pneumonia being mistaken for localised alveolar oedema. However, diagnosis and treatment of HF is started as soon as the patient is admitted 
to hospital and not usually after radiology consult. The Heart Failure Association of the European Society of Cardiology has published a position paper which recommends proper assessment of cardiomegaly, pulmonary venous congestion, pleural effusions, interstitial and alveolar oedema by all admitting physicians [30]. This study, therefore, reflects how CXRs would be interpreted by the majority of clinicians treating patients presenting with breathlessness.

Most CXRs were done within the first $24 \mathrm{~h}$ of admission but the CXR scores of those who had their first film after $24 \mathrm{~h}$ were lower. It is unknown which of these CXR appearances were influenced by diuretic administration. In another study, Kobayashi and colleagues found that residual pulmonary congestion on the CXR prior to discharge predicted a composite outcome of all-cause mortality or rehospitalisation for heart failure at 1 year [31].

\section{Conclusions}

In patients admitted to hospital for HF, there is a high prevalence of radiographic features of congestion. Worsening features of congestion are associated with variables related to worse prognosis. An increase in the CXR score is related to increasing all-cause mortality but is not an independent predictor of outcome when other variables are taken into account.

Acknowledgements The authors would like to thank all patients who participated in this study, without which a deeper understand of what factors relate to outcomes in patients with acute heart failure would not be possible

Author contributions DP, PP and ALC conceived and designed the study. DP, PP, KD, JB, AU, OB, SS and SK acquired the data. DP, PP, ALC and ARR analysed and interpreted the data. DP drafted the manuscript. All the authors critically revised the manuscript for important intellectual content. DP is the guarantor. The corresponding author attests that all the listed authors meet authorship criteria and that no others meeting the criteria have been omitted.

Funding DP, SS and OB are funded by the NIHR. Philips Research provided the income of two research nurses, co-authors KD and JB, for this study.

Availability of data and material Available from corresponding author (DP) upon reasonable request.

\section{Declarations}

Conflict of interest IS and JR are employed by Philips Research. JGFC, ALC, KD and JB have received departmental research support from Philips. ALC has received department support from Novartis and payment for advisory boards from Novartis, Vifor and Pfizer.

Ethics approval Ethical approval from the South Yorkshire Research Ethics Committee (REC ref: 12/YH/0344) and is conducted in accord- ance with ICH-GCP, Declaration of Helsinki, the Data Protection Act 1998 and NHS Act 2006.

Open Access This article is licensed under a Creative Commons Attribution 4.0 International License, which permits use, sharing, adaptation, distribution and reproduction in any medium or format, as long as you give appropriate credit to the original author(s) and the source, provide a link to the Creative Commons licence, and indicate if changes were made. The images or other third party material in this article are included in the article's Creative Commons licence, unless indicated otherwise in a credit line to the material. If material is not included in the article's Creative Commons licence and your intended use is not permitted by statutory regulation or exceeds the permitted use, you will need to obtain permission directly from the copyright holder. To view a copy of this licence, visit http://creativecommons.org/licenses/by/4.0/.

\section{References}

1. Donkor A, McDonagh T, Hardman S (2017) National heart failure audit April 2015 to March 2016. National Institute for Cardiovascular Outcomes Research (NICOR), London

2. Mebazaa A, Yilmaz MB, Levy P et al (2015) Recommendations on pre-hospital \& early hospital management of acute heart failure: a consensus paper from the Heart Failure Association of the European Society of Cardiology, the European Society of Emergency Medicine and the Society of Academic Emergency Medicine. Eur J Heart Fail 17:544-558. https://doi.org/10.1002/ejhf. 289

3. Ponikowski P, Voors AA, Anker SD et al (2016) 2016 ESC Guidelines for the diagnosis and treatment of acute and chronic heart failure. Eur Heart J 37:2129-2200m. https://doi.org/10.1093/eurhe artj/ehw128

4. Danzer CS (1919) The cardio-thoracic ratio: an index of cardiac enlargement. Am J Med Sci 157:513-552

5. Gluecker T, Capasso P, Schnyder P et al (1999) Clinical and radiologic features of pulmonary edema. Radiographics 19:1507-1531. https://doi.org/10.1148/rg.242035075

6. Gheorghiade M, Follath F, Ponikowski P et al (2010) Assessing and grading congestion in acute heart failure: a scientific statement from the Acute Heart Failure Committee of the Heart Failure Association of the European Society of Cardiology and endorsed by the European Society of Intensive Care Medicine. Eur J Heart Fail 12:423-433. https://doi.org/10.1093/eurjhf/hfq045

7. Clark AL, Coats AJS (2000) Unreliability of cardiothoracic ratio as a marker of left ventricular impairment: Comparison with radionuclide ventriculography and echocardiography. Postgrad Med J 76:289-291. https://doi.org/10.1136/pmj.76.895.289

8. Allen CJ, Guha K, Sharma R (2015) How to improve time to diagnosis in acute heart failure-clinical signs and chest X-ray. Card Fail Rev 1(2):69-74. https://doi.org/10.15420/cfr.2015.1.2. 69

9. Petrie MC, McMurray JJV (2003) It cannot be cardiac failure because the heart is not enlarged on the chest $\mathrm{x}$-ray. Eur J Heart Fail 5:117-119. https://doi.org/10.1016/S1388-9842(02)00239-8

10. Roguin A, Behar D, Ben Ami H et al (2000) Long-term prognosis of acute pulmonary oedema-an ominous outcome. Eur J Hear Fail J Work Gr Hear Fail Eur Soc Cardiol 2:137-144. https://doi. org/10.1016/S1388-9842(00)00069-6

11. Battler A, Karliner JS, Higgins C et al (1980) The initial chest $\mathrm{X}$-ray in acute myocardial infarction prediction of early and late mortality and survival. Circ 61(5):1004-1009. https://doi.org/10. 1161/01.cir.61.5.1004 
12. Brezins M, Benari B, Pap V, Cohen A, Bursztein S, Markiewicz W (1993) Left ventricular function in patients with acute myocardial infarction, acute pulmonary edema, and mechanical ventilation: relationship to prognosis. Crit Care Med 21:380-385

13. Lin M, Yang YF, Chiang HT et al (1995) Reappraisal of continuous positive airway pressure therapy in acute cardiogenic pulmonary edema: Short-term results and long-term follow-up. Chest 107:1379-1386. https://doi.org/10.1378/chest.107.5.1379

14. Burch G, DePasquale N (1969) Congestive heart failure-acute pulmonary edema. JAMA 208:1895-1897

15. Packer M (2019) Why are physicians so confused about acute heart failure? N Engl J Med 381:774-776. https://doi.org/10.1056/ nejme 1909053

16. Clark AL, Cleland JGF (2013) Causes and treatment of oedema in patients with heart failure. Nat Rev Cardiol 10:156-170. https:// doi.org/10.1038/nrcardio.2012.191

17. Nikitin NP, Witte KKA, Thackray SDR et al (2003) Effect of age and sex on left atrial morphology and function. Eur J Echocardiogr 4:36-42. https://doi.org/10.1053/euje.2002.0611

18. Shelton RJ, Clark AL, Goode K et al (2006) The diagnostic utility of $\mathrm{N}$-terminal pro-B-type natriuretic peptide for the detection of major structural heart disease in patients with atrial fibrillation. Eur Heart J 27:2353-2361. https://doi.org/10.1093/eurheartj/ ehl233

19. Kabala JE, Wilde $P$ (1987) The measurement of heart size in the antero-posterior chest radiograph. Br J Radiol 60:981-986. https:// doi.org/10.1259/0007-1285-60-718-981

20. Austin PC, Lee DS, D'Agostino RB, Fine JP (2016) Developing points-based risk-scoring systems in the presence of competing risks. Stat Med 35:4056-4072. https://doi.org/10.1002/sim.6994

21. Bartlett JW, Frost C (2008) Reliability, repeatability and reproducibility: analysis of measurement errors in continuous variables. Ultrasound Obstet Gynecol 31:466-475. https://doi.org/10.1002/ uog. 5256

22. Melenovsky V, Andersen MJ, Andress K et al (2015) Lung congestion in chronic heart failure: haemodynamic, clinical, and prognostic implications. Eur J Heart Fail 17:1161-1171. https://doi. org/10.1002/ejhf.417
23. Seah JCY, Tang JSN, Kitchen A et al (2019) Chest radiographs in congestive heart failure: visualizing neural network learning. Radiology 290:514-522. https://doi.org/10.1148/radiol.20181 80887

24. European Commission (2021) European Guidelines on Quality Criteria for Diagnostic Images. https://www.sprmn.pt/pdf/Europ eanGuidelineseur16260.pdf. Accessed 17 Mar 2021

25. Arora S, Patel P, Lahewala S et al (2017) Etiologies, Trends, and Predictors of 30-Day Readmission in Patients With Heart Failure. Am J Cardiol 119:760-769. https://doi.org/10.1016/j.amjca rd.2016.11.022

26. Aggarwal S, Gupta V (2014) Demographic parameters related to 30-day readmission of patients with congestive heart failure: Analysis of 2,536,439 hospitalizations. Int J Cardiol 176:13431344. https://doi.org/10.1016/j.ijcard.2014.07.140

27. Saito M, Negishi K, Marwick TH (2016) Meta-analysis of risks for short-term readmission in patients with heart failure. Am J Cardiol 117:626-632. https://doi.org/10.1016/j.amjcard.2015.11. 048

28. Lourenço P, Ribeiro A, Cunha FM et al (2016) Is there a heart rate paradox in acute heart failure? Int J Cardiol 203:409-414. https:// doi.org/10.1016/j.ijcard.2015.10.129

29. Kobayashi M, Bercker M, Huttin O et al (2019) Chest X-ray quantification of admission lung congestion as a prognostic factor in patients admitted for worsening heart failure from the ICALOR cohort study. Int J Cardiol. https://doi.org/10.1016/j.ijcard.2019. 06.062

30. Mullens W, Damman K, Harjola VP et al (2019) The use of diuretics in heart failure with congestion- a position statement from the Heart Failure Association of the European Society of Cardiology. Eur J Heart Fail 21:137-155. https://doi.org/10.1002/ejhf.1369

31. Kobayashi M, Watanabe M, Coiro S et al (2019) Mid-term prognostic impact of residual pulmonary congestion assessed by radiographic scoring in patients admitted for worsening heart failure. Int J Cardiol 289:91-98. https://doi.org/10.1016/j.ijcard.2019.01. 091 